\title{
Advanced Energy Meter
}

\author{
Sujit Kumar Dash, Tapas Kumar Mohapatra
}

\begin{abstract}
This Paper presents an advanced and remote reading of energy meter. This paper is beneficial taking a meter reading remotely andthere is no need for meter readers to visit each house of customer for collecting the consumed energy data on the monthly basis and to distribute the bill slips. In this advanced technology a microcontroller is installed in the energy meter to monitor and record the meter readings. In case of a client defaulter, no need to send a person of utility to cut-off the customer connection. Utility will interrupt and reconnect the client association by short message service (SMS). Furthermore, the customer can check the status of electricity (load) from anywhere. In this system energy meter readings are being transferred by creating use of GSM.
\end{abstract}

Index Terms--Advanced Energy Meter (AEM), Involuntary Meter Reading (IMR), Global System for Mobile (GSM), Short Messaging Services(SMS).

\section{INTRODUCTION}

The billing of utility system is up till nowobvious in the world as for concern post-paid energy meter. In Asian country, utilities are using a conservative way of electricity billing.An energy meter reader has to go home to home and takes the reading of consumed electricity physically. These readings are brought to utility administration office. The criterion of utility charge is applied consistent with the utility service rules and laws. The employee of the utility goes door to door again and gave the bill slips of the utility to the respective consumer. [1],[2],[3].

Definitely rising energy production costs, continuously expanding interest for environmental friendly and solid advancements, require discerning and economicusage of energy recourses. This makes destinations todevelop a progressed metering and energy control system. Rising energy utilization could be diminished by optimizing the energy production network. This should be possible in many ways: making progressively adaptable and widespread billing, using constant control of structure environmental (heating, air molding and ventilation, lighting, security, and so forth.) and increasing estimation accuracy. There are a few robotized meter perusing (AMR)systems in the market, yet they have one common drawback - they are centered distinctly around information gathering. The new energy metering and control system "Rubisafe Information System" (RIS) created by ABAxis Industries (Kaunas, Lithuania) offers unique opportunity for effective energy utilization management. It gives data about the use of energy distribution arrange. Utilizing this information burden control help to balance the entire network. By getting singular utilization esteems together with suitable valuing assessment, multi-house residential customers can direct their costs for energy consumption. Peak period disappointments can be stayed away from or their impact minimized by utilizing productive and a keen control system. The fundamental pieces of this framework are remotely configurable controllers having savvy load management algorithms. This framework has traffic costs control calculations for information moves. Two-way correspondence (high cost medium) is utilized for moving immense measures of data while essential gadget information and caution messages are sent only one way by utilizing an ease medium (for example single direction traffic, for example, SMS or short radio messages).The organization utilizing this framework can cut their exploitation costs by starting two-way communication when the framework controller is prepared to send the new information orin crisis situations. Information about the framework status and all control functionalities is accessible on the web. Adaptable charging options, readable measurable information, a crisis alert framework and easy control capacities are advertised. Energy providers equipped with such framework, could give hourly meter readings including the remote gadget status, load control, evaluative value data and energy robbery detection. Also, they will ready to guarantee the framework equipment security and approved access, and furthermore nature of administration Detailed load flow are often provided by good energy meters to the shoppers in order that they will accomplish their loading effectively. Advanced energy meter is used to improve the efficiency of the existing energy meter by installing micro controlling unit into the meter. Smart and new energy meter are used for Involuntary Meter Reading (IMR) to extend the accuracy of the meter reading. In present times, there may be error while taking the reading by meter readers so for eliminating these type of fault PLC may be used for viewing the energy data of each and ever. Metering data can be communicatedthrough

Wireless module and Zig-Bee but they are limited to their short range hence for this reason they cannot provide a rate effective solutions to the user. Though, for longer isolated distance a GSM module system is used which is more economical than Wi-Fi andZig-Bee.

Autonomouselectric billing is a uniquesolution to overcome the errors and faults in traditional billing system.sincein traditional billing there is ahuge depletion of time along with the capitals. In advanced and autonomous billing system there is no need of physical metering reading and generating bill slip.
Revised Manuscript Received on September 14, 2019.

Sujit Kumar Dash, Dept. of Electrical \& Electronics Eng., Siksha O Anusandhan Deemed to be University, Odisha, India.(Email: sujitdash@soa.ac.in)

Tapas Kumar Mohapatra, Dept. of Electrical Eng., Siksha O Anusandhan Deemed to be University, Odisha, India.(Email: tapasmohapatra@soa.ac.in) 


\section{ADVANCED ENERGYMETER}

An advanced energy meters and normal conventional meters are same in sizebut advanced energy meters are digital in nature [8]. Advanced Energy Meter deals with more comprehensive readings than Kilowatt hour in order that the generating system can setup the enlargementof grid and improved value of power quality [9],[10],[11]. The Advanced Meter is intended in order that it deals with the voltage and load currents by measuring it and employing sensors for the voltage and current rather than PT and CT transformers and then save the values of obtained voltage and current into integrated controllerand energy meterissue the power calculations respectively[12].

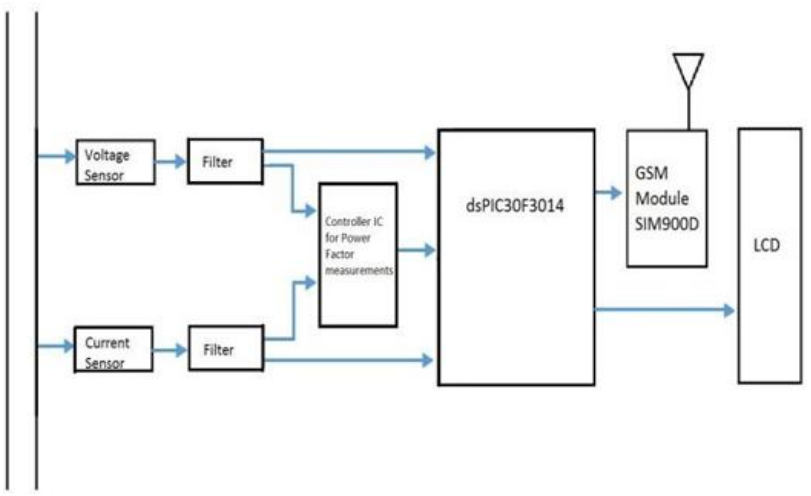

Figure 1. Block diagram of Advanced Energy Meter.

Theproposal of Advanced Energy Meter includes the mensuration ofvoltage and load current via sensors and nourishing them to energy meter integrated circuit that changes it into the real power consumed by the load. Power factor issue is checked by mensuration of shiftingthe phase between current and voltage of load. Microcontroller accomplish the calculations relatedwith power and energy spent and display the reading on the display unit. furthermore because it directs the reading of advancedenergy meter with the assistance of GSM module[13]. Active power,power factor, reactive power, load current,voltage and units (kilowatt-hours) are measured and showed in display unit with success. The Measured meter reading which isdirected from GSM electronic equipment is received on phone with success.

Theadvanced energy meter is use to accomplish a two way communication between the energy meter and gridmanagement furthermore taking of reading by readers of total consumed power can be eliminated if advanced energy meter is applied to house to house and the wastage of capital with time can be reduced. The bill slip generation will also be eliminated by this meter. [14],[15].

The following are the features of advanced energy meter;

- $\quad$ By installing advanced energy meter, the consumer as well as utility can get automatic reading remotely without making an effort.

- $\quad$ The advanced energy meter measures the reading in more advanced form, it displays the consumed energy by customer, voltage, active and reactive power along with a load current.

- In advanced energy meter the administration has an authority to cut off the connection of the defaulter consumers remotely by SMS.

- The Advanced Energy Meter answers to the messages and transmit it back to the consumer whenever needed.

- The main feature of this advanced energy meter is that the Consumer can check his load status remotely from anywhere by simply sending messages by mobile.

\section{METHODOLOGY OF ADVANCED ENERGYMETER}

The GSM module is connected to the utility as well as consumer system whenever they ask for power consumed related data. An antenna is attached to the GSM module to improve the signal strength. Whole system is embedded inside an energy meter box and the meter is unaffected by climatic environmental, it is water resistance. The size of this advanced energy meter is same as conventional energy meter but is more advanced in terms of technology, accuracy and reliability. The main responsibility of advancedenergy meter is to transmit the live information of energy consumed by customer to utility system whenever require. The sensors are also employed in the energy meter to sense the rms value of voltage and current. After sensing, these rms values are transferred to the microcontroller. The micro controller is used to calculate reactive and active power of the consumer's meter. In smart Energy Meter we tend to used sensors to measure voltage and current rather than current and voltage transformers. The reading from Utility administration SMS is being received by smart energy meter programmable interface and therefore the action is performed by the meter in step with provided data.

A major feature of good Energy Meter is that utility company will bring to an end and reconnect the affiliation of energy of any user with the assistance of SMS while not causation the person to perform the task manually [16]. It can be utilized in case when the utility company needs to disconnect a consumer due to non- payment of bills or some other reasons. Another key feature of the advance energy meter is that it has an alarm circuit installed inside, that tells the consumers if they cross the load limit higher than the rated value. [15] In circumstance customer doesn't scale back his load meter mechanically bring to a halt the patron association.

\section{COMPONENTS OF ADVANCED ENERGYMETER}

Advanced energy meter comprises of the following components:

A) Power factor measurement

B) Voltage and current measurement

C) GSM module

\section{A) Power factormeasurement}

The power factor is defined as the phase angle between voltage and current. It dealswith the efficiency of power which is transferred to valuable work. In this experimentit ismeasured by enchanting the waves of voltage and current 
with XOR and assist by microcontroller. The weak sinusoidal waveform is converted into square waveformby LM 358.

It is seen that after performing XOR logic, there exist double frequency. XOR logic is used to combine square voltage and current waveform and resultant waveform is shown by fig.3(c). For fundamental frequency, the $\mathrm{O} / \mathrm{P}$ signal obtained at XOR takes only8 to $9 \mathrm{~ms}$ in case of power factor zero and zeroin case of power factor is 1 . Therefore,for definite value of pf the obtained output of XOR lies between 0 and -1 .
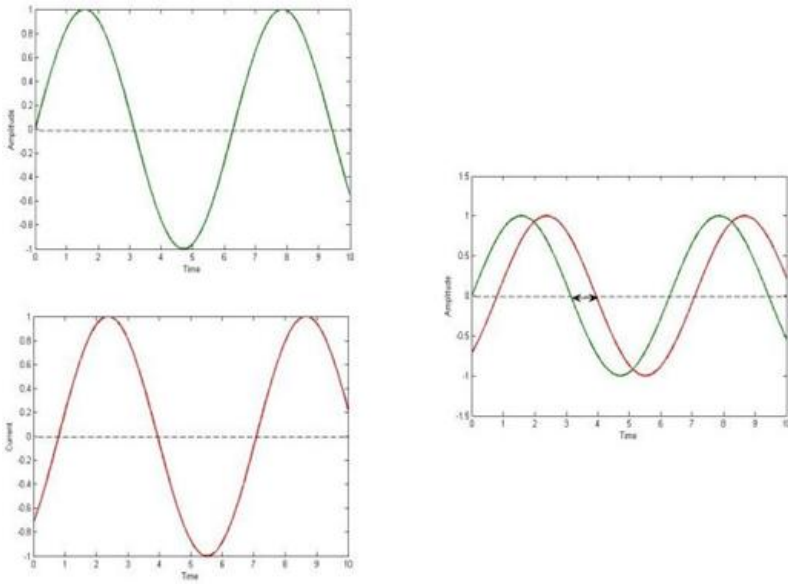

Fig.2(a) Voltage and current waveformFigure2(b) phase shift of current and voltage

B) Voltage and currentmeasurement.

In this experiment the sensors which is embedded inside the meter box is used to measure voltage and load current. ACS71-20A isa current sensor which providesroot mean square value of currents. With the help of current sensor an alternating as well as direct current can be measured easily. The range of measurement of this sensor is up to 20A. The sensitive current are measured by operational amplifier measurements. Small value of current can also be measured by adjusting operational amplifier. The outputcurrent of these sensorshave linear differences with measured currents.
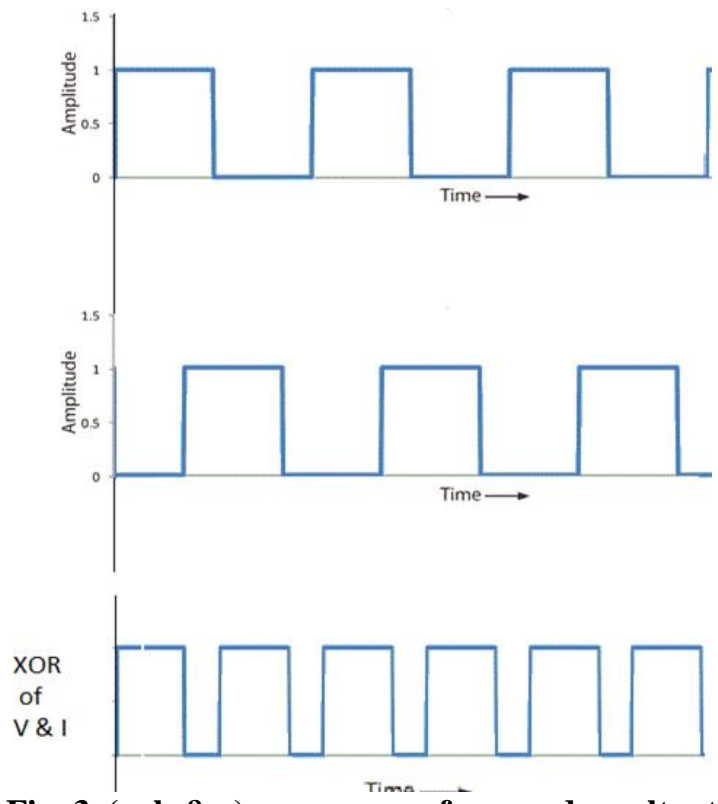

Fig. 3. (a, b \& c).square waveform and resultant waveformof XOR operation

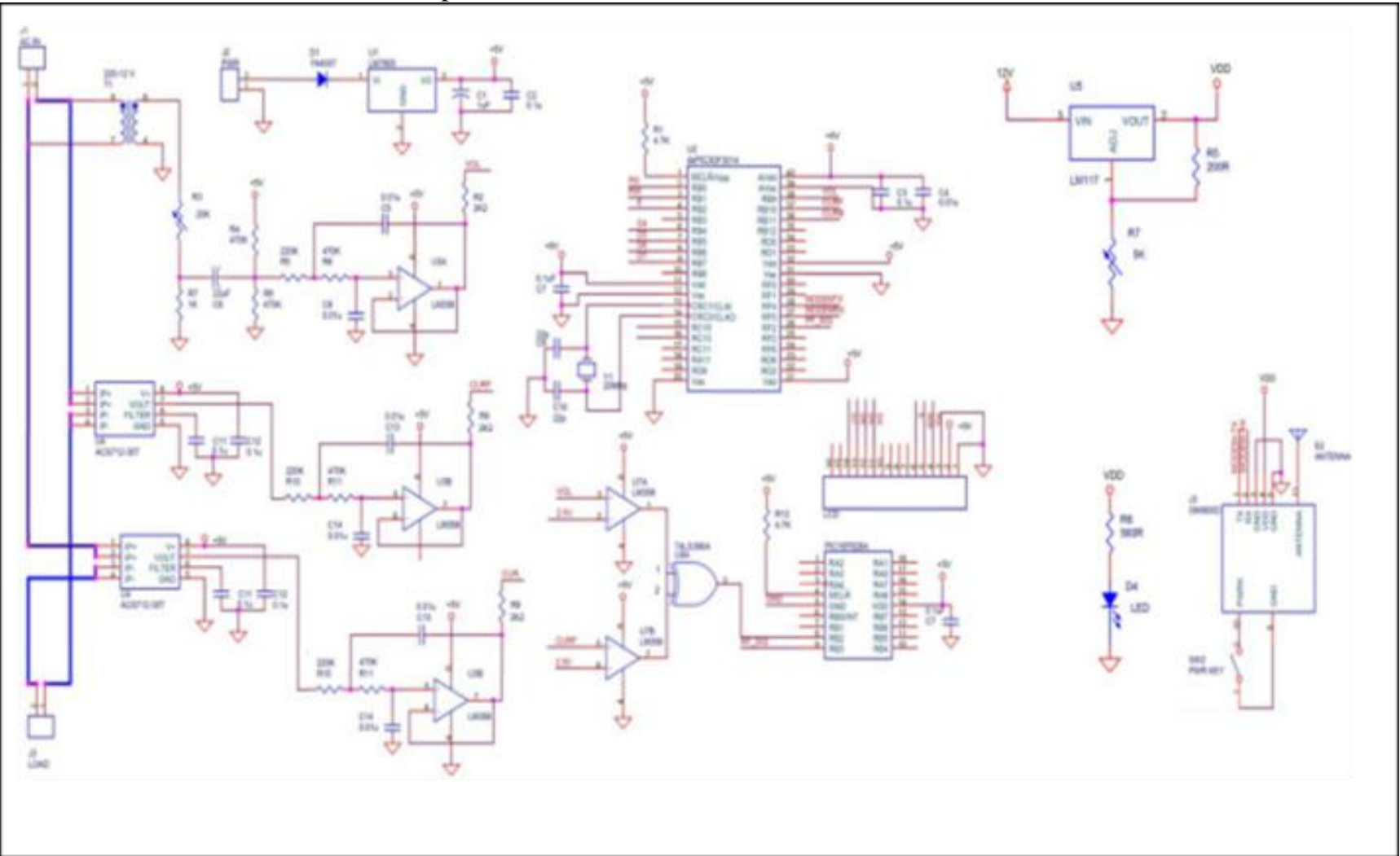

Figure 4: Circuit diagram of Advanced Energy Meter 


\section{c) GSM Module}

The transmission of this system uses GSM module. A GSM module is employed as cell phone gadget / electronic communicatingmodulewhich collects the power consumed data and transmit it to desired phone number [17],[13]. The GSM module used in this experiment is SIM600. A chip or SIM card is employed in the mobile phone for sending and receiving the data. The reading of meter can also be seen by PLC but due to generating unwanted noise by PLC it is not permissible [18],[19]. Metering data can be communicated throughWireless module and Zig-Bee but they are limited to their short range hence for this reason they cannot provide a rate effective solution to the user. Though, for longerdistant the GSMmodule is very much economical.

\section{RESULTS}

The exactness of Advanced Energy Meter is check by comparisons of readings received by SMS which is displayed on the display unit.The effectiveness of Advanced Energy Meter is also seen by switching and detaching the usersmetering connection. The performance of the energy meter is also checked by connecting various load such as $50 \mathrm{~W}, 100 \mathrm{~W}, 200 \mathrm{~W}$ and $1000 \mathrm{~W}$.

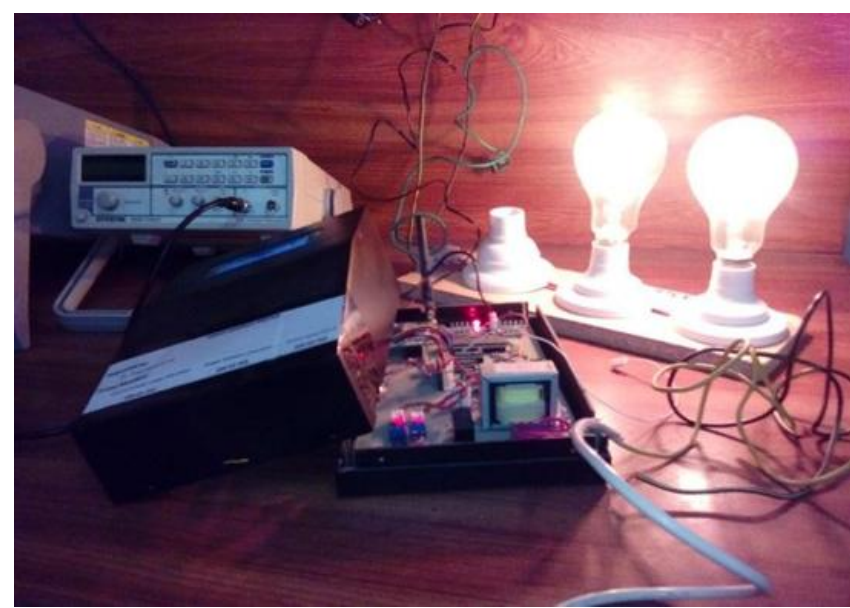

Figure 5: Working of Advanced Energy meter

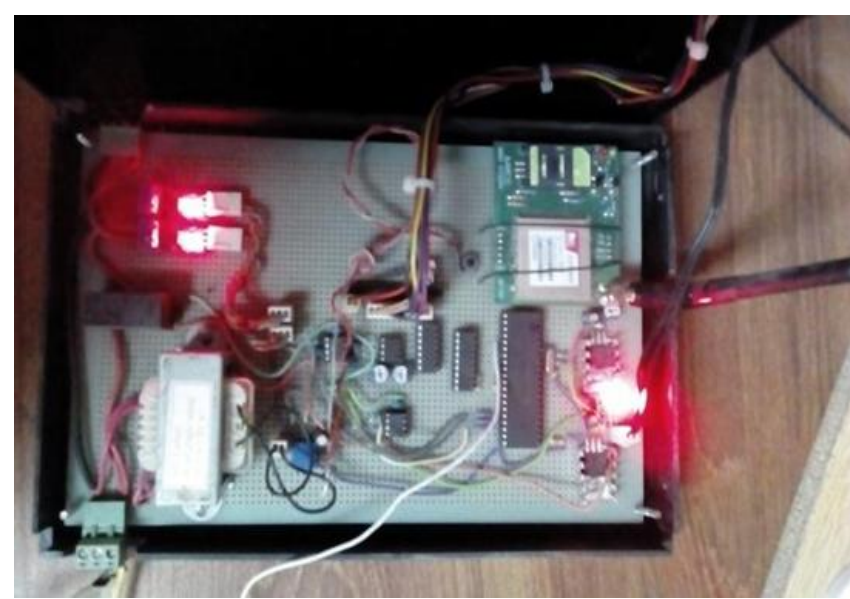

Figure 6: Interior circuit of Advanced Energy Meter.

\section{CONCLUSION}

This paper deals with the study and methodology of Advanced Energy Meter and describes how Advanced Energy Meter is easily applied remotely for taking the energy reading autonomously without making much effort. This meter is foremostinexpensiveapplication to advance humankind during this stage of knowledge. With the growing technology it becomes essential to make the world more autonomous and simpler. This paper conjointly deals with the faulty consumers who don't pay bill and theft the electricity. So for this purpose an advanced energy meter monitor and send the energy consumed data of customer to utility system and atomically cut the supply of defaulter customer thereby eliminating corruption of meter reading.

\section{REFERENCES}

1. V. V. Das, "Wireless communication system for energy meter reading," in ARTCom 2009 - International Conference on Advances in Recent Technologies in Communication and Computing, 2009.

2. C. Liting, T. Jingwen, and Z. Dahang, "Networked remote meter-reading system based on wireless communication technology," in Proceedings of IEEE ICIA 2006 - 2006 IEEE International Conference on Information Acquisition, 2006.

3. K. Ashna and S. N. George, "GSM based automatic energy meter reading system with instant billing," in Proceedings - 2013 IEEE International Multi Conference on Automation, Computing, Control, Communication and Compressed Sensing, iMac4s 2013, 2013.

4. V. Preethi and G. Harish, "Design and implementation of smart energy meter," in Proceedings of the International Conference on Inventive Computation Technologies, ICICT 2016, 2017.

5. M. M. Islam, M. Ahmad, M. A. Islam, A. F. Mitul, M. F. Malek, and M. A. Rashid, "Electronic energy meter with remote monitoring and billing system," in 2012 7th International Conference on Electrical and Computer Engineering, ICECE 2012, 2012.

6. L. Cao, W. Jiang, and Z. Zhang, "Networked wireless meter reading system based on ZigBee technology," in Chinese Control and Decision Conference, 2008, CCDC 2008, 2008.

7. A. H. Primicanta, M. Y. Nayan, and M. Awan, "ZigBeeGSM based automatic meter reading system," in 2010 International Conference on Intelligent and Advanced Systems, ICIAS 2010, 2010.

8. A. Rashdi, R. Malik, S. Rashid, A. Ajmal, and S. Sadiq, "Remote Energy Monitoring, Profiling and Control Through GSM Network,” Arab. J. Sci. Eng., 2013.

9. J. Bhatt and H. K. Verma, "Design and development of wired building automation systems," Energy Build., 2015.

10. T. Chandler, "The technology development of automatic metering and monitoring systems," 2008.

11. M. F. Faisal and A. Mohamed, "A new technique for power quality based environmental monitoring," 2009.

12. P. Rakesh Malhotra and R. Seethalakshmi, "Automatic meter reading and theft control system by using GSM," Int. J. Eng. Technol., 2013.

13. A. C. Tan, C. H. R. Lee, and V. H. Mok, "Automatic power meter reading system using GSM network," Power Engineering Conference, 2007. IPEC 2007. International. 2007.

14. R. Lutolf, "Smart Home concept and the integration of energy meters into a home based system," Seventh International Conference on Metering Apparatus and Tariffs for Electricity Supply 1992. 1992. 
15. A. Abdollahi, M. Dehghani, and N. Zamanzadeh, "SMSbased reconfigurable automatic meter reading system," in Proceedings of the IEEE International Conference on Control Applications, 2007.

16. T. Khalifa, K. Naik, and A. Nayak, "A survey of communication protocols for automatic meter reading applications," IEEE Communications Surveys and Tutorials. 2011

17. Y. Bao and X. Jiang, "Design of electric energy meter for long-distance data information transfers which based upon GPRS technology," in 2009 International Workshop on Intelligent Systems and Applications, ISA 2009, 2009.

18. S. G. Kerk, "An AMR study in an Indian utility," 2008.

19. S. C. Soh and S. G. Kerk, "The electricity and metering trends in Singapore," 2008. 